ceftazidime alone. However, this also results in the development of a rash, this time after only one dose. Which of the following statements are true and which false?

(a) Resolution of symptoms on dechallenge is indicative of a drug aetiology

(b) Occurrence of the reaction much sooner after starting the second antibiotic than on primary exposure is indicative of an immune-mediated adverse reaction

(c) A negative skin test would exclude the drugs as the cause of symptoms

(d) Lack of previous reports of cross-reactivity indicates that the drugs were unlikely to be responsible for the adverse effect

(e) Desensitisation is a reasonable management option in this patient

10 Which of the following statements regarding adverse drug reaction (ADR) reporting by the yellow card system in the UK are true and which false?

(a) ADR reporting is compulsory

(b) All serious ADRs should be reported

(c) ADR reporting provides good ADR incidence data

(d) Doctors, dentists, coroners, pharmacists and nurses are allowed to report on yellow cards

(e) Yellow card reports can be used to establish definitively that a drug has caused a particular reaction

\section{Guidelines on completing the answer sheet for those who wish to submit their answers on paper}

A loose leaf answer sheet is enclosed, which will be marked electronically at the Royal College of Physicians. Answer sheets must be returned by 21 March 2005 to: CME Department (SAQs),

Royal College of Physicians, 11 St Andrews Place, London NW1 4LE.

Overseas members only can fax their answers to 02074874156

Correct answers will be published in the next issue of Clinical Medicine.

*Further details on CME are available from the CME department at the Royal College of Physicians (address above or telephone 02079351174 extension 306 or 309).

Your completed answer sheet will be scanned to enable a quick and accurate analysis of results. To aid this process, please keep the following in mind:

1 Please print your GMC Number firmly and neatly

2 Only write in allocated areas on the form

3 Only use pens with black or dark blue ink

4 For optimum accuracy, ensure printed numbers avoid contact with box edges

5 Please shade circles like this: Not like this: $\$$

6 Please mark any mistakes made like this:

7 Please do not mark any of the black squares on the corners of each page

8 Please fill in your full name and address on the back of the answer sheet in the space provided; this will be used to mail the form back to you after marking.

\title{
CME Infection SAQs \\ Answers to the CME SAQs published in Clinical Medicine November/December 2004
}

$\begin{array}{llllllllll}\text { Q1 } & \text { Q2 } & \text { Q3 } & \text { Q4 } & \text { Q5 } & \text { Q6 } & \text { Q7 } & \text { Q8 } & \text { Q9 } & \text { Q10 } \\ \text { a) } F & \text { a) } F & \text { a) } T & \text { a) } F & \text { a) } F & \text { a) } T & \text { a) } T & \text { a) } F & \text { a) } T & \text { a) } T \\ \text { b) } F & \text { b) } F & \text { b) } F & \text { b) } F & \text { b) } F & \text { b) } F & \text { b) } T & \text { b) } F & \text { b) } F & \text { b) } T \\ \text { c) } F & \text { c) } T & \text { c) } F & \text { c) } F & \text { c) } F & \text { c) } F & \text { c) } T & \text { c) } T & \text { c) } F & \text { c) } F \\ \text { d) } T & \text { d) } T & \text { d) } F & \text { d) } T & \text { d) } F & \text { d) } T & \text { d) } F & \text { d) } F & \text { d) } T & \text { d) } T \\ \text { e) } T & \text { e) } F & \text { e) } F & \text { e) } F & \text { e) } F & \text { e) } F & \text { e) } T & \text { e) } T & \text { e) } T & \text { e) } F\end{array}$

